Jônatas Oliveira

OChttps://orcid. org/0000-0003-2110-5920

Samira Oskinis²

Ohttps://orid.org/0000-0002-1548-6109

Angélica Carreira dos Santos

Ohttps://orcid. org/0000-0002-3937-0258

Táki Athanássios Cordás Dhtps://orcid.org/0000-0003-3929-0175

\section{Existe uma relação entre autocompaixão e adição à comida em mulheres com comportamentos alimentares disfuncionais?}

\author{
Is there a relationship between self-compassion and food \\ addiction in women with dysfunctional eating behaviors?
}

DOI: $10.1590 / 0047-2085000000286$

\section{RESUMO}

Objetivo: Verificar a relação entre autocompaixão e adição à comida em mulheres com comportamento alimentar disfuncional. Métodos: Mulheres de um grupo de apoio completaram as escalas de compulsão alimentar, restrição cognitiva, autocompaixão, Escala de Adição à Comida de Yale versão modificada 2.0 (YFAS 2.0) e questionário de Hay para práticas compensatórias. Foram identificados a prevalência de adição à comida e sintomas segundo a YFAS. Para serem incluídas, as participantes deveriam atingir pontuação para compulsão alimentar e, para análises, foram divididas em função da presença de práticas compensatórias. O grupo foi analisado por meio de testes de correlação de Pearson entre variáveis de interesse, e os grupos bulímico e compulsivo foram comparados com teste $t$ de Student ( $<$ < 0,05; software JASP). Resultados: Participaram do estudo 190 mulheres. De acordo com a YFAS, 95,3\% ( $n=181)$ tinham adição à comida, e os escores da escala apresentaram correlação negativa com a autocompaixão e com a compulsão alimentar ( $p=0,014$ em ambas). Os níveis de autocompaixão apresentaram correlação negativa com as questões \#3, \#5, \#6, \#8 e \#9 da YFAS ( $p<0,05)$. Conclusões: Este estudo traz dados para a discussão da necessidade de analisar como a autocrítica atrelada ao sofrimento de quem apresenta comportamento alimentar disfuncional afeta o preenchimento da escala, trazendo identificação com a noção de vício, já que esta é culturalmente aceita.

\section{PALAVRAS-CHAVE}

Adição à comida, autocompaixão, transtornos alimentares, comportamento alimentar, compulsão alimentar.

\section{ABSTRACT}

Objective: To verify the relationship between self-compassion and food addiction in women with disordered eating behavior. Methods: Women from a support group completed the binge eating scale, cognitive restraint, self-compassion, modified Yale Food Addiction Scale 2.0 (YFAS 2.0), and Hay's questionnaire for compensatory practices. The prevalence of "food addiction" and symptoms, according to YFAS, was identified. To be included, participants should achieve binge eating scores, and for analysis, they were divided according to the presence of compensatory practices. The group was analyzed using Pearson's correlation tests between variables of interest, and the bulimic and compulsive groups were compared with Student's t-test ( $p<0.05$; JASP software). Results: 190 women participated in the study. According to YFAS, 95.3\% $(n=181)$ had food addiction, and the scale scores showed a negative correlation with self-compassion and binge eating ( $p=0.014$ for both). The levels of self-compassion showed a negative correlation with questions \#3, \#5, \#6, \#8 and \#9 of YFAS ( $p<0.05)$.

Conclusions: This study brings data to discuss the need to analyze how self-judgment linked to the suffering of the experience of those who show upset eating behavior, affects the scale filling, bringing identification with the notion of addiction since it is culturally accepted.

KEYWORDS

Food addiction, self-compassion, eating disorders, eating behavior, binge eating. 


\section{INTRODUÇÃO}

Os transtornos mentais, em sua maioria, apresentam uma caraterística marcante de autocrítica, que se caracteriza pelo reconhecimento de qualidades e defeitos'. Werner e cols.' revisaram detalhadamente a associação entre autocrítica e transtornos mentais, mostrando relações positivas com sintomas de transtornos alimentares (TAs), transtorno de ansiedade e transtornos de personalidade, bem como com problemas interpessoais.

Os TAs, em específico, contêm uma série de componentes clínicos e comportamentais que requerem manejo especíico com avaliação crítica que leve em conta a autoavaliação que oscila entre negação e exageros como, por exemplo, a negação da gravidade presente no quadro de anorexia nervosa $(\mathrm{AN})^{2}$ e relatos de exageros alimentares ou consumos alimentares julgados inapropriadamente como "compulsões" pelos indivíduos com o transtorno ${ }^{3}$. Além da própria psicopatologia do TA, que inclui uma atitude negativa perante o self, os transtornos depressivos e/ou ansiosos e os transtornos de personalidade comórbidos podem influenciar tanto a gravidade dos sintomas como a autoavaliação sobre eles ${ }^{4}$.

Considerando essa relação, o conceito de autocompaixão traz novas perspectivas para o entendimento de como as atitudes perante o self podem contribuir para sintomas e manutenção da psicopatologia ${ }^{5,6}$. Definida por Neff como uma atitude positiva direcionada ao self, a autocompaixão compreende três mecanismos com estrutura de seis fatores. Propõem-se um estado e práticas de atenção plena no lugar de superidentificação com sintomas e sofrimento (primeiro mecanismo). A autogentileza é proposta como um estado que pode levar a comportamentos positivos - inclusive na alimentação - quando o indivíduo compreende e desenvolve atitudes que evitam a autocrítica severa (segundo mecanismo). Por fim, compreender o sofrimento e os acontecimentos inesperados - erros, sintomas e doenças - como parte da humanidade abre espaço para uma visão compartiIhada que evita o isolamento (pensamentos e sentimentos), de tal modo que a humanidade comum é sobreposta ao isolamento (terceiro mecanismo) ${ }^{7}$.

A superidentificação é um estado envolvido em intenso julgamento e depreciação do self, exemplificado por Kristin Neff: "Quando estou para baixo, tendo a ficar obsessivo e me fixar em tudo o que está errado"7,8. Terapias focadas em autocompaixão, por exemplo, são baseadas na relação entre a culpa e a manutenção da psicopatologia nos TAs ${ }^{5}$. Os indivíduos tornam-se mais compassivos após esse tratamento, estando abertos às inadequações pessoais, falhas e sofrimento, respondendo a eles com humanidade comum, atenção plena e autogentileza7. A consciência de erros, falhas e questões que envolvem sofrimento sem, no entanto, negá-las (desenvolvimento da atenção plena com diminuição da autocrítica) abre espaço para a entrada de possibilidade de estratégias que envolvam a autogentileza e o autocuidado, mesmo durante sentimentos ou eventos negativos, quando normalmente esses pacientes tendem a realizar mais restrições, ter episódios de comer emocional, compulsão alimen$\operatorname{tar}(\mathrm{CA})$ ou práticas compensatórias ${ }^{8-10}$.

Alguns autores vêm postulando que a perda de controle, o consumo alimentar, apesar das consequências negativas, e o comprometimento social na alimentação se dão por um padrão comportamental e alterações neurais semelhantes aos do transtorno por uso de substâncias ${ }^{11}$. Assim, a chamada "adição à comida" vem ganhando espaço na literatura com base no arcabouço científico emprestado de teorias da dependência química ${ }^{12}$ e de resultados em modelos animais. Em 2009, foi desenvolvida a Escala de Adição à Comida de Yale (YFAS), que consiste em um questionário baseado no autorrelato para fornecer diagnóstico e sintomas de adição à comida ou, também comumente chamada, comer aditivo (addictive-like eating)11,13,14. Apesar da discussão polarizada entre prós e contras ao conceito, os indivíduos com uma relação conturbada com a comida utilizam culturalmente o termo há muito tempo ${ }^{15}$. Assim, a aceitação cultural do termo vem proporcionando a disseminação dele antes mesmo de sua caracterização como possível construto.

Os TAs são marcados por tentativas malsucedidas de controle sobre a alimentação, com restrições alimentares severas, longos jejuns e outros comportamentos direcionados para a perda de peso. No estudo de Gearhardt et al. ${ }^{16}$, os maiores escores na YFAS estavam associados ao excesso de peso precoce e ao histórico do início de dietas restritivas. Foi demonstrada a relação entre a gravidade do transtorno de compulsão alimentar (TCA), urgência negativa e dificuldade na regulação emocional em indivíduos com adição à comida ${ }^{17}$. Ainda, os indivíduos com TCA e coocorrência de adição à comida $(41,5 \%)$ apresentaram maior gravidade em sintomas depressivos, psicopatologia alimentar e autoestima em relação aos indivíduos que não atingiram a classificação ${ }^{18}$.

Palmeira e cols. demonstraram que a autodepreciação nos TAs ocorria, em parte, pela inabilidade em ser autocompassivo ${ }^{19}$. Considerando a influência da autocompaixão sobre os TAs $^{20}$ e a noção de gravidade atribuída aos indivíduos com TA e coocorrência de adição à comida, este estudo teve como objetivo investigar se há relação entre autocompaixão e adição à comida em mulheres com comportamento alimentar disfuncional.

\section{MÉTODOS}

Este estudo teve caráter exploratório, quantitativo e transversal, e a pesquisa foi conduzida com mulheres de um grupo de apoio fechado no Facebook que tem por objetivo compartiIhar experiências de ajuda mútua para o tratamento dos TAs. 
As coletas ocorreram durante o segundo semestre de 2018 com o auxílio das administradoras do grupo. Após o contato inicial com as administradoras do grupo, os questionários foram disponibilizados para as participantes, garantindo o anonimato e o contato com os pesquisadores responsáveis em caso de necessidade. O questionário continha opção de lembrete ao final da página para quando alguma questão deixasse de ser respondida, evitando, assim, a perda de dados. 0 consentimento informado foi obtido por todos os indivíduos que participaram da pesquisa, e as participantes só completavam o questionário on-line caso estivessem de acordo com o Termo de Compromisso Livre e Esclarecido utilizado em formato eletrônico, no qual assinalavam a concordância ou não em participar do estudo. Este estudo aprovado pelo Comitê de Ética da Faculdade de Medicina da Universidade de São Paulo (número de aprovação: 2.695.532).

\section{Critério de inclusão e exclusão}

Primeiramente, procedeu-se ao cálculo dos escores para CA, sendo incluídas nas análises apenas as participantes com pontuação maior que 17 (presença de CA) ${ }^{21}$. Como critério de exclusão, foram removidas as participantes com pontuação menor que 17, assim como as que atingiram classificação de baixo peso segundo o índice de massa corpórea (IMC $\leq 18,5$ $\mathrm{kg} / \mathrm{m}^{2}$ ). Essa remoção foi embasada no critério A para AN (baixo peso sustentado por restrição alimentar) segundo o Manual Diagnóstico e Estatístico de Transtornos Mentais (DSM-5)².

\section{INSTRUMENTOS}

Todos os instrumentos foram transcritos para um questionário on-line. Informações sobre as características das participantes (idade, peso, altura), estado civil e escolaridade, assim como o autorrelato do histórico para diagnóstico de TA e histórico de tratamento, foram coletadas por meio de um questionário estruturado.

\section{Escala de Compulsão Alimentar (BES)}

Para avaliação da presença de CA, foi utilizada a BES, que consiste em 64 afirmações do tipo "Eu tenho o hábito de engolir minha comida sem realmente mastigá-la. Quando isso acontece, em geral me sinto desconfortavelmente empanturrado(a) por ter comido demais". Desenvolvida por Gormally e cols. ${ }^{22}$, a BES é amplamente utilizada para avaliar a presença de CA e apresenta pontuação de corte no escore 17, sendo os valores entre 18 e 26 pontos considerados relativos à presença de CA de gravidade média, e a pontuação maior que 26 caracteriza CA grave 22 . $O$ instrumento foi traduzido e posteriormente validado para o português brasileiro e comparado com a entrevista semiestruturada para
TCA (Structured Clinical Interview - SCID), com pontuação de corte confirmada para a população brasileira, demonstrando sensibilidade de 97,9\% e a confiabilidade teste-reteste segundo coeficiente kappa $(0,65)$ e kappa ponderado de 0,66, com alfa de Cronbach de 0,89. Foi considerada adequada para população brasileira ${ }^{23}$. $O$ alpha de Cronbach calculado para esta amostra foi de $0,89^{23}$.

\section{Restrição cognitiva}

Para avaliar o nível de restrição cognitiva, foi utilizada a subescala de restrição cognitiva da The Three Factor Eating Questionnaire. O questionário foi primeiramente desenvolvido por Stunkard e Messick como medida de estudo para restrição, alimentação emocional e descontrole alimentar ${ }^{24}$. Teve sua versão reduzida para 21 itens. A subescala de restrição cognitiva consiste em seis questões com pontuação Likert que tratam de algumas crenças e comportamentos direcionados à perda de peso ou à mudança da forma corporal, como disposto na questão: "Eu conscientemente me controlo nas refeições para evitar ganhar peso". No Brasil, teve sua versão traduzida para o português e posteriormente validada ${ }^{25}$, apresentando consistência interna por meio do alpha de Cronbach igual a 0,83.

\section{Escala de Autocompaixão}

Para avaliação dos níveis de autocompaixão, foi utilizada a Escala de Autocompaixão, desenvolvida por Neff'. Caracteriza-se por um instrumento de 26 itens em formato Likert de cinco pontos, com respostas que vão de "quase nunca" a "quase sempre". Além da pontuação total, ela fornece seis fatores que compreendem as subescalas: atenção plena, superidentificação, autogentileza, autocrítica, humanidade comum e isolamento. $\mathrm{O}$ instrumento foi adaptado e validado para o português brasileiro e apresentou resultados adequados, e a análise fatorial confirmatória da escala endossou a estrutura de seis fatores, conforme a versão original ${ }^{26}$. 0 alpha de Cronbach calculado para a amostra do presente estudo foi de 0,92.

\section{Escala de Adição à Comida de Yale versão modificada 2.0 (mYFAS 2.0)}

Para avaliação da pontuação total e presença de sintomas de adição à comida, foi utilizada a versão reduzida da Escala de

Adição à Comida de Yale $^{14}$. $\mathrm{O}$ instrumento foi desenvolvido com base nos critérios diagnósticos para transtorno por uso de substâncias em sua primeira versão, segundo os critérios do DSM-IV, sendo publicada a primeira versão em $2009^{11}$. Teve sua versão atualizada com 13 itens (versão 2.0) três anos após as atualizações do DSM-5, em 2013. O questionário acessa comportamento e cognições relacionadas com a dificuldade em controlar o consumo de alguns alimentos no último ano, e sua pontuação fornece um escore contínuo, 
opções de categorização e divisão em sintomas. No Brasil, a versão reduzida foi recentemente adaptada para o português brasileiro e apresentou resultados psicométricos aceitáveis ${ }^{27}$. O alpha de Cronbach calculado para esta amostra foi de 0,86 . Neste estudo, foram calculadas as pontuações totais e porcentagem de sintomas.

\section{Questionário de Hay}

Foi desenvolvido por Hay para investigar a frequência de comportamentos alimentares inapropriados, como a CA, práticas compensatórias inapropriadas e prática de dieta restritiva nos últimos três meses ${ }^{28}$. A frequência de respostas desse questionário são "nunca", "menos de uma vez por semana", "uma vez por semana", "duas ou mais vezes por semana". Neste estudo, foi utilizada a versão adaptada para o português brasileiro, que apresentou bons valores de confiabilidade (kappa de 0,92 no estudo de validação) ${ }^{29}$, considerando que a frequência desses comportamentos nos últimos três meses associa-se com os diagnósticos de bulimia nervosa (BN) ou TCA (ausência de comportamentos compensatórios), segundo o DSM-52. Neste estudo, a presença de comportamentos compensatórios foi utilizada para categorização em grupo específico segundo a descrição da próxima seção.

\section{Divisão de grupos}

Os grupos foram divididos de acordo com a presença de práticas compensatórias inapropriadas extraídas do questionário de Hay. Assim, considerando a presença de CA segundo a pontuação na BES, aqueles que assinalaram "nunca" na questão de práticas compensatórias foram alocados no grupo compulsivo, e os que apresentaram práticas compensatórias foram alocados no grupo bulímico. Essa divisão foi baseada nos sintomas descritos no DSM-5 para BN (CA associada com práticas compensatórias) e TCA (presença de CA sem a ocorrência de práticas compensatórias)².

\section{Análise dos dados}

Os dados foram expressos em valores médios, desvio-padrão (DP), valores mínimos e máximos. O IMC foi calculado segundo a fórmula peso/altura². A normalidade das variáveis foi analisada considerando os parâmetros de assimetria e curtose de até 2,0 e até 7,0, respectivamente, para verificar distorções na distribuição dos dados, conforme critérios sugeridos por Kim³ ${ }^{30}$ Após essa análise, procedeu-se à execução de testes paramétricos para comparação dos resultados médios entre grupos (teste t de Student) e foram calculados os tamanhos de efeito das diferenças entre grupos por meio do cálculo de d de Cohen (<0,19: insignificante; 0,2-0,49: pequeno; 0,5-0,79: moderado; e <0,8: grande). Foram realizadas análises de correlação de Pearson entre os escores totais da Escala de Autocompaixão (e subescalas) com as 13 questões da YFAS. Considerou-se nível de significância de 5\% com análises conduzidas no software JASP.

\section{RESULTADOS}

Participaram do estudo 190 mulheres com 26 anos em média (DP =6,22; com variação de 17 a 48 anos) e 75,54 kg em média (DP = 19,14; com variação de 47 a 150 kg; 9 mulheres não souberam relatar o peso). A categoria de Eutrofia teve $32,63 \%$ ( $n=62)$, a categoria sobrepeso, 33,68\% ( $n=64)$, a categoria Obesidade grau I, 15,79\% ( $n=30)$ e as categorias Obesidade grau II e Obesidade grau III tiveram 13,16\% ( $n=$ 25). Dados sobre escolaridade demonstraram, em toda a amostra, que 59\% ( $n=112)$ têm o ensino superior (>15 anos de educação), 39\% ( $n=74$ ) terminaram os estudos no ensino médio (11 a 14 anos de educação) e uma minoria (2\%, n=4) completou o ensino fundamental (8 a 10 anos de educação). A maioria se declarou solteira $(66,7 \%, n=127)$, enquanto $31,5 \%(n=60)$ eram casadas e $3 \%(n=2)$, divorciadas. Em relação à orientação sexual, $79,5 \%(n=151)$ eram heterossexuais, $16,8 \%(n=32)$ bissexuais, 3\% homossexuais $(n=2)$ e 4 participantes assinalaram a opção "prefiro não relatar".

O autorrelato de histórico para diagnóstico de TA foi majoritariamente para TCA $(65,79 \% ; n=125)$, seguido de BN $(21,58 \% ; n=41)$ e AN (5,79\%; $n=11)$. Pouco mais da metade passou por tratamento para TA $(53,68 \% ; n=102)$, e o restante, não. Segundo a divisão realizada de acordo com o questionário de Hay, 93 participantes (48,9\% em relação ao total) reportaram práticas compensatórias (grupo bulímico), e 97 participantes (51,1\% em relação ao total) reportaram ausência desses comportamentos (grupo compulsivo). No total avaliado, 95,3\% ( $n=181)$ atingiram pontuação para adição à comida (Tabela 1).

\section{Comportamento alimentar disfuncional nos grupos bulímico $(n=93)$ e grupo compulsivo $(n=97)$}

O grupo bulímico apresentou maior gravidade na CA segundo a BES (t: 2,576, $p=0,011$, d: 0,374), maiores níveis de restrição cognitiva (t: 3,226, p=0,001, d: 0,468) e pontuação total na YFAS (t: 3,320, para $=0,001$, d: 0,482) em relação ao grupo compulsivo (com pequenos tamanhos de efeito, segundo $d$ de Cohen). Em relação aos indicativos de autocompaixão, o grupo compulsivo apresentou maiores pontuações tanto no escore total (t: -2,802, $p=0,006, d$ : -0,407) quanto nas subescalas. A subescala isolamento foi a única medida que não variou entre os grupos (t: - 0,428, p=0,669, d: -0,062) (Tabela 2). Em relação aos escores totais na Escala de Autocompaixão, foram encontradas correlações negativas (análise da amostra total, $n=190)$ com YFAS ( $p=0,014)$, escores de CA ( $p=0,014$ em ambos), assim como correlações negativas com a pontuação média das questões \#3, \#5, \#6, \#8 e \#9 da YFAS (Tabela 3). 
Tabela 2. Comparações de escores de compulsão alimentar, restrição cognitiva e adição à comida, em função da presença ou não de práticas compensatórias

\begin{tabular}{|c|c|c|c|c|}
\hline & $\begin{array}{l}\text { Grupo bulímico } \\
\qquad(\mathrm{n}=93)\end{array}$ & $\begin{array}{l}\text { Grupo compulsivo } \\
(\mathrm{n}=97)\end{array}$ & \multirow[t]{2}{*}{ Valor de $\mathrm{p}$} & \multirow[t]{2}{*}{ d (Cohen) } \\
\hline & Média (DP) & Média (DP) & & \\
\hline Compulsão alimentar & $32,27(6,97)$ & $29,74(6,58)$ & 0,011 & 0,37 \\
\hline Restrição cognitiva & $16,88(3,91)$ & $15,01(4,10)$ & 0,001 & 0,46 \\
\hline YFAS & $55,90(18,50)$ & $47,49(16,29)$ & 0,001 & 0,48 \\
\hline Autocompaixão & $51,77(14,89)$ & $58,16(16,51)$ & 0,006 & $-0,40$ \\
\hline SJ & $7,79(3,14)$ & $9,14(3,66)^{\mathrm{a}}$ & 0,007 & $-0,39$ \\
\hline $\mathrm{Ol}$ & $5,92(2,29)$ & $6,89(2,69)$ & 0,009 & $-0,38$ \\
\hline $\mathrm{CH}$ & $9,17(3,51)$ & $10,36(3,74)$ & 0,025 & $-0,32$ \\
\hline I & $9,79(2,38)$ & $9,94(2,52)$ & 0,669 & $-0,06$ \\
\hline SK & $9,71(4,0)$ & $10,66(3,94)$ & 0,017 & $-0,34$ \\
\hline$M$ & $9,37(3,63)$ & $10,66(3,94)$ & 0,020 & $-0,34$ \\
\hline
\end{tabular}

Teste t de Student; a = significância no teste de Levene indicando que as variâncias não são iguais; DP: desvio-padrão; d: tamanho do efeito da diferença expresso por meio do d de Cohen; Escala de Adição à Comida de Yale - Versão 2.0 (YFAS 2.0), Atenção plena (M), Superidentificação (OV), Autogentileza (SK), Autocrítica (SJ), Humanidade comum (CH) e Isolamento (I).

Nota: graus de liberdade para as comparações entre grupos: 188.

Tabela 1. Sintomas acessados pela Escala de Adição à Comida na amostra total $(n=190)$

\begin{tabular}{lcc}
\hline & n & \% \\
\hline 1) Substância usada por um tempo maior que o previsto & 172 & 90,5 \\
2) Desejo persistente ou repetidas tentativas malsucedidas em parar & 184 & 96,8 \\
3) Muito tempo/atividade para obter, usar e se recuperar & 186 & 97,9 \\
4) Atividades sociais, ocupacionais ou recreacionais importantes deixadas ou reduzidas & 132 \\
5) Uso apesar do conhecimento das consequências adversas & 179 \\
6) Tolerância (aumento acentuado na quantidade e acentuada diminuição do efeito) & 161 \\
7) Sintomas característicos da abstinência; substância consumida para alívio da retirada & 94,5 \\
8) Uso persistente apesar de problemas sociais ou interpessoais & 164 \\
9) Falha no cumprimento de obrigações principais (no trabalho, escola ou lar) & 153 \\
10) Uso em situações fisicamente perigosas & 84,7 \\
11) Desejo intenso, ou uma urgência para consumo & 145 \\
12) Uso causa prejuízo ou angústia clinicamente significativos & 80,3 \\
\hline
\end{tabular}

Tabela 3. Correlações com escores totais para autocompaixão $(n=190)$

\begin{tabular}{|c|c|}
\hline & $\begin{array}{l}\mathrm{r} \text { de Pearson } \\
\text { (valor de } p \text { ) }\end{array}$ \\
\hline Compulsão alimentar & $-0,18(0,014)$ \\
\hline YFAS (2.0) & $-0,18(0,014)$ \\
\hline \#3 Eu evitei o trabalho, escola ou atividades sociais porque eu tive medo que eu fosse comer demais lá. & $-0,23(0,001)$ \\
\hline \#5 0 meu comportamento alimentar me causou muito sofrimento. & $-0,30(<0,001)$ \\
\hline $\begin{array}{l}\text { \#6 Eu tive problemas significativos na minha vida por causa de comida. Podem ter sido problemas com a minha rotina diária, trabalho, } \\
\text { escola, amigos, família, ou de saúde. }\end{array}$ & $-0,24(<0,001)$ \\
\hline \#8 Eu continuei comendo da mesma forma, mesmo este fato tendo me causado problemas emocionais. & $-0,17(0,022)$ \\
\hline \#9 Comer a mesma quantidade de alimento não me deu tanto prazer como costumava me dar. & $-0,15(0,041)$ \\
\hline
\end{tabular}

Escala de Adição à Comida de Yale - Versão 2.0 (YFAS), Atenção plena (M), Superidentificação (OV), Autogentileza (SK), Autocrítica (SJ), Humanidade comum (CH) e Isolamento (I); Questões \#3, \#5, \#6, \#8 e \#9 descritas no material suplementar de Nunes-Neto et al.27.

Nota: Apenas dados $p<0,05$ apresentados. 


\section{DISCUSSÃO}

Este é o primeiro estudo brasileiro que verificou a prevalência de adição à comida em mulheres com CA. Os presentes resultados demonstram que a autocompaixão está negativamente associada com afirmações e pontuação total para adição à comida. Assim, considerando o sofrimento alimentar e a visão negativa do self nos indivíduos com comportamento alimentar disfunciona| ${ }^{31}$, sugere-se uma influência no processo de autorrelato das questões alimentares que a YFAS apresenta. De acordo com a YFAS, quase todas as participantes (95,3\%) atingiram pontuação para adição à comida. No estudo da adaptação da escala para o português brasileiro realizado com 7.639 participantes (71,3\% mulheres), a prevalência encontrada foi de $4,31 \%{ }^{27}$.

Quando as participantes foram comparadas em função da presença de práticas compensatórias (Tabela 2), notaram-se maiores níveis de CA, restrição cognitiva e pontuação total na YFAS (grupo bulímico) em comparação ao grupo compulsivo. Com relação ao fato de a obesidade ser um fator associado à adição à comida ${ }^{13,16}$, é importante ressaltar que as categorias de Obesidade foram representadas pela minoria das participantes (Obesidade grau I em 15,79\%, $n=$ 30; e Obesidade graus II e III em $13,16 \%, n=25$ ) e que as participantes do grupo com baixo peso foram removidas do estudo. Guertin e cols. demonstraram que falar ou tratar frequentemente de assuntos sobre estar gordo foi positivamente associado com desmotivação e alimentação não saudável, enquanto a autocompaixão foi positivamente associada com melhores comportamentos alimentares ${ }^{32}$.

As questões da YFAS são precedidas de um cabeçalho que trata dos hábitos alimentares no último ano e da "dificuldade em controlar o consumo", que, no caso dos TAs, possivelmente atinge as atitudes alimentares voltadas ao controle, proibições e crenças sobre efeitos dos alimentos no corpo (autocrítica possivelmente associada a crenças distorcidas). Abaixo foram transcritas as informações do cabeçalho da versão brasileira27:

"Esta pesquisa pergunta sobre seus hábitos alimentares no último ano. As pessoas às vezes têm dificuldade em controlar o quanto elas comem de certos alimentos, tais como:

\footnotetext{
- Doces como sorvete, chocolate, biscoito, bolo, doce - Amidos como pão, massa e arroz

- Salgados, como batata frita e biscoito

- Alimentos gordurosos, como carne, bacon, hambúrguer, pizza e batata frita
}

- Bebidas açucaradas, como refrigerante e bebida energética

Quando as questões seguintes perguntam sobre 'Certos alimentos', por favor, pense em quaisquer alimentos ou bebidas semelhantes aos listados nos grupos de alimentos ou bebidas acima ou quaisquer outros alimentos que você teve dificuldade de controlar o consumo no último $a^{27} 0^{27}$

Tem-se que a adição à comida avaliada pelos sintomas descritos na YFAS pode ser um preditor da gravidade do TCA ${ }^{33}$. Gearhardt e cols. ${ }^{18}$ observaram que o número de sintomas do YFAS mostrou correlação positiva com altos escores de sintomas depressivos, baixa autoestima, dificuldade na regulação emocional e maior frequência de episódios de comer compulsivo. No estudo de Kelly e Carter, a autocrítica foi preditora para níveis agravados na psicopatologia de pacientes com TA, e os pacientes com menor medo da autocompaixão apresentaram menor psicopatologia do TA ${ }^{34}$. No presente estudo, o escore total de autocompaixão apresentou correlação negativa com escores de CA ( $p=0,014$; tabela 3). Os dados corroboram os de Barata-Santos e cols., que mostraram que a compaixão pelo próprio corpo foi negativamente associada à $\mathrm{CA}^{35}$.

\section{Análises de correlação entre autocompaixão e questões da Escala de Adição à Comida de Yale (mYFAS 2.0)}

Na questão \#3 “Eu evitei o trabalho, escola ou atividades sociais porque eu tive medo que eu fosse comer demais lá" (correlação negativa com autocompaixão, $p=$ 0,001) (Tabela 3), é importante questionar o "medo" que esses indivíduos teriam em frequentar alguns locais $^{36}$. Indivíduos que apresentam CA têm altos níveis de restrição alimentar, o que é um grande determinante para a perda de controle ${ }^{37}$. O medo de comer faz parte das cognições presentes no TA, que envolvem uma série de regras alimentares e crenças a respeito de quantidades e "efeitos fisiológicos" que o consumo alimentar proporciona, portanto deve-se compreender o "medo" do qual a escala trata com essas ressalvas ${ }^{38}$.

$\mathrm{Na}$ questão \#5 “O meu comportamento alimentar me causou muito sofrimento" (Tabela 3), encontrou-se correlação negativa com o escore total de autocompaixão $(p<0,001)$. Considerando novamente as instruções da YFAS, quando se pede que o indivíduo responda em relação a uma classe de alimentos (palatáveis, doces, refrigerantes), pensar sobre a relação com esses alimentos não necessariamente reflete o consumo habitual do indivíduo, mas possivelmente evoca o sofrimento dessa relação. Indivíduos com AN, que sustentam a restrição alimentar prolongada, atingiram classificação de adição à comida (69,2\%) segundo a escala $a^{39}$ e apresentam o medo de comer em excesso ${ }^{40,41}$.

A questão \#6 “Eu tive problemas significativos na minha vida por causa de comida. Podem ter sido problemas com a minha rotina diária, trabalho, escola, amigos, família, ou de saúde" (Tabela 3) apresentou correlação com o escore total ( $p<0,001)$. Essa questão abre 
outro debate importante para a área, pois os problemas e o comprometimento social que ocorrem no TA não poderiam se resumir ao "objeto comida", pois não é a comida que causa o TA nem uma classe de alimentos específicos. Foi demonstrado em estudo randomizado que a autocompaixão reduziu comportamentos do TA, e a coocorrência de adição à comida com os diagnósticos de BN ou TCA indicou a severidade da patologia alimentar ${ }^{42}$.

O comer compulsivo reduz a percepção da quantidade de alimentos ingeridos. No entanto, a autocompaixão pode ajudar o indivíduo a lidar de maneira proativa com seus sentimentos, regulando o consumo ${ }^{43}$. Em outro estudo, a subescala autogentileza foi preditora de 37,6\% da variância de sintomas de TA ${ }^{44}$. O desenvolvimento de um comer compassivo requer olhar com autogentileza para as escolhas alimentares, abrindo espaço para um dos objetivos para a remissão da CA durante o tratamento dos TAs, que é a diminuição da restrição alimentar ${ }^{5,31,34}$.

O grupo bulímico, além de apresentar maior gravidade na compulsão, atingiu menores valores na subescala de atenção plena em relação ao grupo compulsivo (com ausência de práticas compensatórias) $(p=0,020)$. Meule e cols. demonstraram correlações entre os escores para YFAS e a preocupação com o peso e com a forma corporal em bulímicas $^{45}$, evidenciando como as questões corporais e sua relação com as crenças alimentares disfuncionais podem estar ligadas a um mecanismo de superidentificação. Na análise de sintomas da YFAS, 69,5\% ( $n=132)$ atingiram pontuação para o sintoma de "redução ou desistência de atividades sociais, ocupacionais e recreacionais", enquanto o menor sintoma presente na amostra foi o "consumo em situações de risco" $(42,1 \% ; n=80)$ (Tabela 1). Os resultados de prevalência total de adição à comida $(95,3 \%)$ devem ser interpretados com cautela em relação aos dados disponíveis para TCA $(41,5 \%)^{18}$ e BN $(100 \%)^{45}$, pois, no presente estudo, a prevalência se refere a um grupo com presença de comportamentos alimentares disfuncionais, e não aos diagnósticos de TA.

A questão \#8 “Eu continuei comendo da mesma forma, mesmo este fato tendo me causado problemas emocionais" (Tabela 3) apresentou correlação negativa com o escore total de autocompaixão $(p=0,022)$ e parece evocar o estado de autocrítica, como visto no discurso do campo da substância química aplicado ao comportamento alimentar quando se fala do comer "apesar das consequências negativas" ${ }^{16}$. Mantzios e cols. ${ }^{47}$ concluíram que isolamento e superidentificação foram associados positivamente com o consumo de açúcar e gordura. O estado de atenção plena que visa diminuir a superidentificação com os problemas alimentares, como tratado na questão \#8, pode minimizar potencialmente as reações automáticas e desatentas em relação à comida, além de reduzir os gatilhos emocionais que levam ao comer emocional e ao consumo alimentar excessivo ${ }^{48}$.
Pelo conhecimento dos autores, o presente estudo foi o segundo na literatura que apresentou a relação entre autocompaixão e adição à comida, sendo o primeiro, de Rainey e cols. ${ }^{49}$, o de que a autocompaixão se configurou como fator protetor para adição à comida. Propomos que as questões da YFAS discutidas apresentam diversos desdobramentos para a compreensão da relação entre os temas, como (1) gravidade do comportamento alimentar, (2) percepção e autorrelato dos sintomas e (3) superidentificação com as cognições e pensamentos propostos pelas questões da YFAS.

\section{Limitações do estudo}

Alguns fatores são limitantes para a discussão e os achados deste estudo. Deve-se considerar que todos os dados foram coletados por meio de autorrelato, sem o uso de entrevistas presenciais. Deve-se considerar a ausência de um diagnóstico formalizado para TA, indicando que os dados devem ser interpretados com cautela para comparações com os quadros de BN e TCA. A grande prevalência de comportamentos alimentares disfuncionais está relacionada com as características do grupo on-line que realiza uma triagem para concentrar mulheres que estão com sintomas recorrentes, e isso culminou na dificuldade em formar um grupo controle (sem (A), sendo possível apenas avaliar os grupos em função da presença ou não de práticas compensatórias.

\section{CONCLUSÕES}

Destaca-se a alta prevalência de adição à comida na amostra (95,3\%) e como as questões da YFAS se baseiam numa autoavaliação acerca da relação dificultosa que se tem com uma classe de alimentos, o que pode ser chamado de "vício" por aqueles que vivenciam um processo psicológico de privação alimentar, pensamentos intrusivos sobre comida e desejo intenso, além da eventual perda de controle. Uma metanálise demonstrou que a autocompaixão foi positivamente associada a comportamentos que promovem saúde ${ }^{50}$. A autocrítica apresentou relação inversa com a pontuação total para adição à comida. Portanto, é necessário analisar como a autocrítica atrelada ao sofrimento da vivência dos comportamentos disfuncionais afeta o preenchimento da escala, trazendo identificação com a noção de "vício", por ser culturalmente aceita.

\section{CONTRIBUIÇÕES INDIVIDUAIS}

Jônatas Oliveira - Contribuiu com a concepção do projeto, a análise, a interpretação dos dados e a redação.

Samira Oskinis - Contribuiu com a interpretação dos dados e a redação. 
Angélica Carreira dos Santos - Contribuiu com a interpretação dos dados e a análise crítica.

Táki Athanássios Cordás - Contribuiu com a concepção do projeto, a supervisão e a aprovação da versão final. Todos os autores aprovaram a versão final do manuscrito.

\section{CONFLITO DE INTERESSES E SUPORTE FINANCEIRO}

Não há.

\section{REFERÊNCIAS}

1. Werner AM, Tibubos AN, Rohrmann S, Reiss N. The clinical trait self-criticism and its relation to psychopathology: A systematic review - Update. J Affect Disord. 2018;246:530-47.

2. American Psychiatric Association (APA). Diagnostic and Statistical Manual of Mental Disorders. Arlington: APA; 2013.

3. Milos G, Kuenzli C, Soelch CM, Schumacher S, Moergeli H, Mueller-Pfeiffer C. How much should I eat? Estimation of meal portions in anorexia nervosa. Appetite. 2013:63:42-7.

4. Culbert KM, Racine SE, Klump KL. Research Review: What we have learned about the causes of eating disorders - A synthesis of sociocultural, psychological, and biological research. J Child Psychol Psychiatry. 2015;56(11):1141-64.

5. Kelly AC, Carter JC, Borairi S. Are improvements in shame and self-compassion early in eating disorders treatment associated with better patient outcomes? Int I Eat Disord 2014:47(1):54-64

6. Taylor MB, Daiss S, Krietsch K. Associations among self-compassion, mindful eating, eating disorder symptomatology, and body mass index in college students. Transl Issues Psychol Sci. 2015;1(3):229-38.

7. Neff KD. The development and validation of a scale to measure self-compassion. Self Identity. 2003;2(3):223-50.

8. Neff KD, Vonk R. Self-compassion versus global self-esteem: two different ways of relating to oneself. J Pers. 2009;77(1):23-50

9. Montero-Marín J, Gaete J, Demarzo M, Rodero B, Lopez LC, García-Campayo J. Selfcriticism: a measure of uncompassionate behaviors toward the self, based on the negative components of the self-compassion scale. Front Psychol. 2016;7:1281.

10. Salvo V, Kristeller J, Montero Marin J, Sanudo A, Lourenço BH, Schveitzer MC, et al. Mindfulness as a complementary intervention in the treatment of overweight and obesity in primary health care: study protocol for a randomised controlled trial. Trials. 2018;19(1):277.

11. Gearhardt AN, Corbin WR, Brownell KD. Preliminary validation of the Yale Food Addiction Scale. Appetite. 2009;52(2):430-6.

12. Avena NM, Rada P, Hoebel BG. Evidence for sugar addiction: Behavioral and neurochemical effects of intermittent, excessive sugar intake. Neurosci Biobehav Rev. 2008;32(1):20-39.

13. Meule A, Gearhardt AN. Five years of the Yale Food Addiction Scale: Taking stock and moving forward. Curr Addict Rep. 2014;1:193-205.

14. Gearhardt AN, Corbin WR, Brownell KD. Development of the Yale Food Addiction Scale Version 2.0. Psychol Addict Behav. 2016;30(1):113-21

15. Meule A. Back by Popular Demand: A Narrative Review on the History of Food Addiction Research. Yale J Biol Med. 2015;88(3):295-302.

16. Gearhardt AN, White MA, Masheb RM, Grilo CM. An examination of food addiction in a racially diverse sample of obese patients with binge eating disorder in primary care settings. Compr Psychiatry. 2013;54(5):500-5.

17. Wolz I, Granero R, Fernández-Aranda F. A comprehensive model of food addiction in patients with binge-eating symptomatology: The essential role of negative urgency. Compr Psychiatry. 2017;74:118-24.
18. Gearhardt AN, White MA, Masheb RM, Morgan PT, Crosby RD, Grilo CM. An examination of the food addiction construct in obese patients with binge eating disorder. Int J Eat Disord. 2012:45(5):657-63

19. Palmeira L, Pinto-Gouveia J, Cunha M. The role of self-disgust in eating psychopathology in overweight and obesity: Can self-compassion be useful? J Health Psychol. 2019;24(13):1807-16

20. Stutts $L A$, Blomquist KK. The moderating role of self-compassion on weight and shape concerns and eating pathology: A longitudinal study. Int J Eat Disord. 2018:51(8):879-89.

21. Marcus MD, Wing RR, Lamparski DM. Binge eating and dietary restraint in obese patients. Addict Behav. 1985:10(2):163-8

22. Gormally JIM, Black S, Daston S, Rardin D. the Assessment of Binge Eating Severity Among Obese Persons. Addict Behav. 1982;7(1):47-55.

23. Freitas S, Lopes CS, Coutinho W, Appolinario JC. Tradução e adaptação para o português da Escala de Compulsão Alimentar Periódica. Rev Bras Psiquiatr. 2001;23(4):215-20.

24. Stunkard AJ, Messick S. The three-factor eating questionnaire to measure dietary restraint, disinhibition and hunger. J Psychosom Res. 1985;29(1):71-83

25. Medeiros ACQ, Yamamoto ME, Pedrosa LFC, Hutz CS. The Brazilian version of the threefactor eating questionnaire-R21: psychometric evaluation and scoring pattern. Eat Weight Disord. 2017;22(1):169-75

26. Souza LK, Hutz CS. Adaptation of the self-compassion scale for use in Brazil: evidences of construct validity. Temas Psicol 2016;24(1):159-72.

27. Nunes-Neto PR, Kohler CA, Schuch FB, Quevedo J, Solmi M, Murru A, et al. Psychometric properties of the modified Yale Food Addiction Scale 2.0 in a large Brazilian sample. Rev Bras Psiquiatr. 2018;40(4):444-8

28. Hay P. The epidemiology of eating disorder behaviors: an Australian community-based survey. Int J Eat Disord. 1998;23(4):371-82.

29. Ferreira JES, Veiga GV. Confiabilidade (teste-reteste) de um questionário simplificado para triagem de adolescentes com comportamentos de risco para transtornos alimentares em estudos epidemiológicos. Rev Bras Epidemiol. 2008;11:393-401.

30. Kim HY. Statistical notes for clinical researchers: assessing normal distribution (2) using skewness and kurtosis. Restor Dent Endod. 2013;38(1):52-4

31. Kelly AC, Carter JC. Eating disorder subtypes differ in their rates of psychosocia improvement over treatment. J Eat Disord. 2014;2(2)

32. Guertin C, Barbeau K, Pelletier L. Examining fat talk and self-compassion as distinct motivational processes in women's eating regulation: A self-determination theory perspective. J Health Psychol. 2018;1359105318781943.

33. Davis C. From passive overeating to "food addiction": a spectrum of compulsion and severity. ISRN Obes. 2013;2013:435027.

34. Kelly AC, Carter JC. Why self-critical patients present with more severe eating disorder pathology: the mediating role of shame. Br J Clin Psychol. 2013;52(2):148-61.

35. Barata-Santos M, Marta-Simões J, Ferreira C. Body compassion safeguards against the impact of major life events on binge eating. Appetite. 2019;134:34-9.

36. Bou Khalil R, Bou-Orm IR, Tabet Y, Souaiby L, Azouri H. Disgust and fear: common emotions between eating and phobic disorders. Eat Weight Disord. 2020;25(1):79-86.

37. Greeno CG, Wing RR, Shiffman S. Binge antecedents in obese women with and without binge eating disorder. J Consult Clin Psychol. 2000;68(1):95-102.

38. Haynos AF, Berg KC, Cao L, Crosby RD, Lavender JM, Utzinger LM, et al. Trajectories of higher- and lower-order dimensions of negative and positive affect relative to restrictive eating in anorexia nervosa. J Abnorm Psychol. 2017;126(5):495-505.

39. Granero R, Hilker I, Agüera Z, Jiménez-Murcia S, Sauchelli S, Islam MA, et al. Food addiction in a Spanish sample of eating disorders: DSM-5 diagnostic subtype differentiation and validation data. Eur Eat Disord Rev. 2014;22(6):389-96.

40. Murray SB, Strober M, Craske MG, Griffiths S, Levinson CA, Strigo IA. Fear as a translationa mechanism in the psychopathology of anorexia nervosa. Neurosci Biobehav Rev. 2018;95:383-95

41. Murray HB, Tabri N, Thomas JJ, Herzog DB, Franko DL, Eddy KT. Will I get fat? 22-year weight trajectories of individuals with eating disorders. Int J Eat Disord. 2017;50(7):739-47.

42. Gearhardt AN, Boswell RG, White MA. The association of "food addiction" with disordered eating and body mass index. Eating Behav. 2014;15(3):427-33. 
43. Mantzios M, Wilson JC. Mindfulness, eating behaviours, and obesity: a review and reflection on current findings. Curr Obes Rep. 2015;4(1):141-6.

44. Ferreira C, Matos M, Duarte C, Pinto-Gouveia J. Shame memories and eating psychopathology: the buffering effect of self-compassion. Eur Eat Disord Rev. 2014;22(6):487-94.

45. Meule A, Von Rezori V, Blechert J. Food addiction and bulimia nervosa. Eur Eat Disord Rev. 2014;22(5):331-7.

46. Parylak SL, Koob GF, Zorrilla EP. The dark side of food addiction. Physiol Behav. 2011;104(1):149-56.
47. Mantzios M, Egan H, Hussain M, Keyte R, Bahia H. Mindfulness, self-compassion, and mindful eating in relation to fat and sugar consumption: an exploratory investigation. Eat Weight Disord. 2018;23(6):833-40.

48. Bal U, Çelikbaş Z, Batmaz S. Association of mindfulness and impulsivity with obesity. Arch Clin Psychiatry (São Paulo). 2018;45(5):130-4.

49. Rainey JC, Furman CR, Gearhardt AN. Food addiction among sexual minorities. Appetite. 2018;120:16-22.

50. Sirois FM, Kitner R, Hirsch JK. Self-compassion, affect, and health-promoting behaviors. Health Psychol. 2015;34(6):661-9. 